\title{
The Cause of Increased Effectiveness of Water-In-Oil Emulsion Over Water Application of Trichoderma Ovalisporum on Theobroma Cacao
}

\author{
Ronald T Collins ${ }^{1 *}$, Mary J Camp² ${ }^{2}$ Bryan A Bailey ${ }^{2}$ and Lyndel W Meinhardt ${ }^{4}$ \\ ${ }^{1}$ US Department of Agriculture, Agriculture Research Service, Sustainable Perennial Crops Laboratory, USA \\ ${ }^{2}$ US Department of Agriculture, Agriculture Research Service, Sustainable Perennial Crops Laboratory, USA \\ ${ }^{3}$ US Department of Agriculture, Agriculture Research Service, Sustainable Perennial Crops Laboratory, USA \\ ${ }^{4}$ US Department of Agriculture, Agriculture Research Service, Sustainable Perennial Crops Laboratory, USA
}

*Corresponding author: Ronald T Collins, Agronomist, US Department of Agriculture, Agriculture Research Service, Sustainable Perennial Crops Laboratory, 10300 Baltimore Ave./ Bldg 001, Rm. 223/Beltsville, MD 20705, USA

Received Date: September 30, 2019

Published Date: October 04, 2019

\begin{abstract}
Experiments in Costa Rica showed that Trichoderma ovalisporum applied in a water-in-oil (corn oil) emulsion gave significantly better control of frosty pod rot disease on cacao (Theobroma cacao) pods when compared to water applications. Experiments were conducted in Ecuador in 2011 and 2012, to determine whether the effectiveness of the T. ovalisporum was supported by the applied spray characteristics (Volume Median Diameter, number of droplets per $\mathrm{cm}^{2}$, droplet volume per $\mathrm{cm}^{2}$, area coverage per $\mathrm{cm}^{2}$, Relative Span Factor and estimated spores per $\mathrm{cm}^{2}$ ) as opposed to the creation of an environment conducive to spore germination and colonization of the cacao pod. The relative span factor and number of deposits were not different between the two formulations. The Volume Medium Diameter, droplet volume, Droplet volume and area coverage were statistically larger for the water formulation than water-in-oil emulsion formulation but largely proportional to the $50 \%$ water content in the water-in-oil emulsion formulation. From the deposit volume, the estimated spore count per $\mathrm{mm}^{2}$ was 1100 for the water formulation and 904 for the water-in-oil emulsion formulation. The spray characteristics of the two formulations did not account for the significantly better control of frosty pod rot by the water-in-oil emulsion. The improved microclimate provided by the water-in-oil emulsion was the primary contributor to the formulation superior performance in the field.
\end{abstract}

Keywords: Corn oil; Emulsion; Trichoderma; Trichoderma ovalisporum; Backpack mist-blower; Droplet analysis

\section{Introduction}

In 2009 and 2010 experiments were conducted in Costa Rica to determine the effectiveness of two different Trichoderma species (Trichoderma ovalisporum and T. harzianum) in different formulations to control frosty pod rot and black pod rot on cacao. The formulations were $0.3 \mathrm{ml} \mathrm{L}^{-1}$ of the surfactant BreakThru 100SL(BT), a mixture of $1 \% \mathrm{w} / \mathrm{v}$ Sure-Jell (source of pectin) and $1 \% \mathrm{w} / \mathrm{v}$ potato dextrose broth (PDB) (PP), and an water-in-oil emulsion composed of $50 \% \mathrm{v} / \mathrm{v}$ corn oil emulsified with $5 \% \mathrm{v} / \mathrm{v}$ lecithin and $50 \%$ PDB. The best treatment was the water-in-oil (corn oil) emulsion containing the biocontrol organism DIS-70a (Trichoderma ovalisporum) with 30.7 percent healthy pods. The water control had 9.9 percent healthy pods. The spray volume was $180 \mathrm{~mL}$ per tree [1].
The purpose of this study is to determine whether the increase in effectiveness of the Trichoderma ovalisporum in the water-in-oil invert emulsion versus water is the result of a difference in spray characteristics of the formulation application in contrast to the creation of a microenvironment by the water-in-oil emersion that is beneficial to spore germination and colonization on the cacao pod. Other researchers have found that water-in-oil emulsions increase the effectiveness of biocontrol agents by altering their surrounding microenvironment. Boyette found in greenhouse research that the application of Colletotrichum truncatum in a water-in-oil emulsion increased the bioherbicidal activity by increasing the time allowed between inoculation and dew onset thus decreasing the dew period length required for spore germination [2]. Boyette also found in field 
research that aqueous applications of Colletotrichum truncatum were not effective in controlling hemp sesbania while applications of unrefined corn oil and surfactant were. He hypothesized that the corn oil protected the conidia from drying out during the dew free period [3]. Mbarga applied Trichoderma asperellum in different formulations to Theobroma cacao pods and then challenges the pods with Phytophthora megakarya. The oil-in-water emulsion had the best control of the disease [4].

Experiments were conducted in 2011 and 2012 at the Tropical Research Experiment Station in Pichilingue, Ecuador to study the spray deposition of water vs. water-in-oil emulsion applied to cacao trees (Theobroma cacao). The goal was to determine if spray characteristics of the water-in-oil invert formulation may contribute to its increased effectiveness.

\section{Methods and Materials}

In the experiments, two formulations were used: water (WC) and water-in-oil (corn oil) emulsion (WIOC). The treatments were applied with a conventional backpack sprayer with $180 \mathrm{~mL}$ of the formulation applied per tree. The treatments were applied with a Stihl SR 420 motorized mist-blower (Andreas Stihl Ltd) fitted with a 1.0 restrictor. For both treatment applications, a setting of 4 was used, correlating to an application rate of $21.2 \mathrm{~mL}$ per second, which was delivered for approximately 8.5 seconds per tree. The conventional applications utilized a gravity feed mechanism to provide the solution to the pump and the angle of application was no more than 15 degrees, to minimize any variability due to the gravity feed.

\section{Formulation of Spray Solutions}

In Costa Rica, the 50/50 water-in-oil invert (WIOC) was prepared by adding lecithin $(6 \% \mathrm{v} / \mathrm{v})$ slowly to a bucket containing corn oil while blending with a stirring rod on an electric drill. Once the ingredients of the oil emulsion were mixed, the emulsion was blended for an additional $5 \mathrm{~min}$. The PDB (1\% w/v) was added to the corn oil/lecithin emulsion, with continual stirring for $5 \mathrm{~min}$. This method was the best available method for producing these formulations due to the large-scale field application that was being conducted.

In the current study the following methodology was used to make the WIOC. First the corn oil (purchased locally) was emulsified with lecithin ( 6 percent $\mathrm{v} / \mathrm{v}$ ). The $500 \mathrm{~mL}$ of corn oil was decanted into a Waring laboratory variable speed blender. The blender was operated at 5000 RPM. The lecithin was added slowly to the blender. After the lecithin was completely decanted, the blending was continued for an additional 5 minutes. After blending the corn oil emulsion, $500 \mathrm{~mL}$ of the PDB (1\% w/v) solution was added. One-third of the PDB was added to the blender, and then pulsed for

Table 1: Treatment Means and Mean Comparisons.

\begin{tabular}{|c|c|c|c|c|c|}
\hline Treatment & VMD & Coverage & Deposits & Deposition \\
\hline WOIC & $151.92 \mathrm{a}^{[\mathrm{a}]}$ & $17.35 \mathrm{a}$ & $714.04 \mathrm{a}$ & $0.73 \mathrm{a}$ \\
\hline WC & $195.81 \mathrm{a}$ & $20.42 \mathrm{a}$ & $724.13 \mathrm{a}$ & $1.09 \mathrm{a}$ \\
\hline
\end{tabular}

${ }^{[a] T r e a t m e n t ~ m e a n s ~ w i t h ~ d i f f e r e n t ~ l e t t e r s ~ a r e ~ s t a t i s t i c a l l y ~ d i f f e r e n t ~ a t ~}<0.05$ significance level.

Citation: Ronald T Collins, Mary J Camp, Bryan A Bailey, Lyndel W Meinhardt. The Cause of Increased Effectiveness of Water-In-Oil Emulsion Over Water Application of Trichoderma Ovalisporum on Theobroma Cacao. World J Agri \& Soil Sci. 3(3): 2019. WJASS.MS.ID.000563. DOI: 10.33552/WJASS.2019.03.000563.
2 seconds at 2000 RPM. The second third of the PDB was added to the blender and pulsed for 2 seconds at 2000 RPM. The remaining PDB was added to the blender and pulsed for 3 seconds at 2000 RPM. The water-in-oil emulsion was then ready for application. The invert was stable for approximately three hours. The water droplets are formed within the WIOC by the shear of the blender's blades at the interface of the aqueous and oil phases during high speed rotation [5]. Because the spray deposition was being analyzed, Trichoderma spores were not added to the spray solution. Rather, the numbers of spores $\mathrm{cm}^{-2} \mathrm{mcL}$ were estimated by multiplying the mcL of deposit volume $\mathrm{cm}^{-2}$ by 103 spores per mcL.

\section{Droplet Analysis}

The spray application was documented utilizing Syngenta water sensitive cards $(5.1 \mathrm{~cm}$ by $7.6 \mathrm{~cm})$. Six cards were attached to leaves of each cacao tree at a height of $137 \mathrm{~cm}$ and equally spaced around the circumference of the tree. The cards were recovered, dried and scanned. Three random locations were selected on each card and analyzed with DepositScan [6]. Each location was analyzed for DV5 (volume medium diameter (VMD); half the volume is in drops of greater diameter), DV10 (10\% of the volume is in drops of greater diameter), DV90 (90\% of the spray volume is in drops of smaller diameter), percentage coverage $\mathrm{cm}^{-2}$, number of drops $\mathrm{cm}^{-2}$ (Deposits), volume $\mathrm{mcL} \mathrm{cm}^{-2}$ (Deposition) and Relative Span Factor (RSF): A dimensionless parameter indicative of the uniformity of the drop size distribution (RSF = (DV90-DV10)/DV5 [7]). The closer this number is to zero, the more uniform the spray application will be. The data was also analyzed to determine if a spread factor was necessary for WOIC. The spread factor was estimated by: Spread factor WOIC $=[$ Deposition $($ WOIC) $/ 90] /[$ Deposition (WC) / 180].

\section{Experimental Design}

An experimental unit consisted of one cacao tree. Each treatment had four replicates. Six water sensitive cards were used per tree. Spray application began at card 1 location. The trees were located at random within an eight-year-old cacao orchard. Three locations on each water sensitive cards were analyzed. A completely randomized design was utilized.

The variables VMD - Relative Span Factor were each analyzed separately as two-factor linear models using PROC MIXED [8] with Treatment and Experiment as the factors, but with no Exp $\mathrm{x}$ Trt interaction. The assumptions of the model were checked and met. As Exp was never statistically significant, Exp was ignored and the variables were analyzed as t-tests using PROC TTEST. The means comparisons are listed in Table 1. The means were not different for any of the measured variables.

\section{Results and Discussions}

\section{Statistical analysis}


Since the WOIC Deposition (0.73) exceeded one half the WC Depositions (1.10), a spread factor was calculated for the WOIC formulation (Table 1). The estimated spread factor for WOIC was $[0.7 / 90] /[1.1 / 180]=1.3$. The Deposit analysis was corrected for the spread factor.

\section{Statistical analysis (adjusted for spread factor)}

Because cards are sub-samples on the experimental unit Tree, the averaged values/tree was used in the analyses. The variables DV5 - Relative Span were each analyzed separately as two-factor mixed models using PROC MIXED (SAS Institute) with Treatment as the fixed factor and Experiment the random. The assumptions of the model were checked and met. For Deposits one value (Exp 2, Trt 2, Tree 4, Deposits $=258.167$ ) showed up as a possible outlier, so the analyses were done without outliers. Analysis of variance results are given in Table 2. Means comparisons are in Table 3.

Table 2: Analysis of Variance ( $p$-value).

\begin{tabular}{|c|c|c|c|c|c|c|}
\hline Source & DF & VMD & Coverage & Deposits & Deposition & Relative Span Factor \\
\hline Treatment & 1 & 0.0265 & 0.0125 & 0.1717 & 0.0095 & 0.6566 \\
\hline
\end{tabular}

[a]Treatment means with different letters are statistically different at $<0.05$ significance level.

Table 3: Treatment Means and Mean Comparisons.

\begin{tabular}{|c|c|c|c|c|c|}
\hline Treatment & VMD & Coverage & Deposits & Deposition \\
\hline WOIC & $130.58 \mathrm{~b}^{[\mathrm{a}]}$ & $12.35 \mathrm{~b}$ & $589.10 \mathrm{a}$ & $0.45 \mathrm{~b}$ \\
\hline WC & $195.81 \mathrm{a}$ & $20.42 \mathrm{a}$ & $724.12 \mathrm{a}$ & $1.10 \mathrm{a}$ \\
\hline
\end{tabular}

[a]Treatment means with different letters are statistically different at < 0.05 significance level.

\section{Comparison of WC and WOIC}

VMD, Coverage and Deposition were all significantly different between the WC and WOIC treatments (Table 3). This is not unexpected because using equivalent application volumes the WC treatment applies twice as much water as the WOIC (180 vs. 90 $\mathrm{mL}$ per tree). The RSF and Deposits were not different between WC and WOIC (Table 3). The RSF describes the uniformity of the spray droplet size distribution around the VMD generated by the backpack sprayer. The non-significant difference between WC (0.94) and WOIC (0.91) RSF indicated that spray droplet formation was similar in both WC and WOIC. If the water phase within the oil phase were not uniform the WOIC's RSF would be much larger than the WC. The Deposits were not statistically different between WOIC and WC (589 vs. 724) (Table 3). Yet, there were about 23\% more WC Deposits than the WOIC. This may be the result of at least two factors. Some of the WOIC droplets may have consisted of oil only and did not register on the water sensitive cards and therefore cannot be estimated. Second, even though the WOIC droplet was large enough to be measured by DepositScan, the water portion of the droplet was not large enough to be measured. Essentially, after accounting for the spread factor WOIC droplets must be twice threshold to be measured by DepositScan.

The Deposits and the number of spores in the droplets are important for the application of biocontrol agents. The WC averaged a Deposition of $1.1 \mathrm{mcL} \mathrm{cm}^{-2}$ and theoretically would contain approximately 1100 spores $\mathrm{cm}^{-2}$. The WOIC averaged a deposition of $0.45 \mathrm{mcL} \mathrm{cm}^{-2}$ for the water portion and would be expected to contain approximately 904 spores per $\mathrm{cm}^{-2}$, assuming spores show preference for water. The characteristics of the spray deposition do not explain the superior protection of Trichoderma ovalisporum in WOIC vs. WC. Taking these factors into account, there is clearly no negative effect on spray deposition due to the WOIC formulation compared to the WC formulation.
Previous research supports the hypothesis that the water-in-oil emulsion creates a beneficial environment for spore germination and tissue colonization because of the oil slowing the droplets water evaporation. This maintains a microenvironment supporting germination of Trichoderma ovalisporum spores and supports colonization of the surface of cacao pods and other cacao tissues. (Crozier et al, 2015) conducted humidity chamber studies with $T$. ovalisporum spores and various formulations including the WOIC and WC. Twenty mcL drops of T. ovalisporum spores $\left(10^{6}\right.$ conidia $\mathrm{mL}^{-1}$ ) were incubated in microwells at $25{ }^{\circ} \mathrm{C}$ and $100 \%$ relative humidity for $15 \mathrm{~h}$. Trichoderma ovalisporum spores in the WOIC formulation achieved $93.6 \%$ germination while germination in the WC formulation was $0 \%$ [1].

\section{Discussion}

The question addressed in this study was do the spray deposition characteristics account for the superior performance of T. ovalisporum in managing frosty pod rot when applied in a WOIC formulation. Although the volume of water in the WIOC was halve that of the $\mathrm{WC}$, the number of deposits were not statistically different, while the VMD, deposit volume, coverage and number of spores were significantly less than that found for WC. Where differences occurred, the spray characteristics correlate with the reduced volume of water in the WIOC formulation. The droplet analysis also showed that water-in-oil emulsion droplets were uniformly formed. Formulation spray characteristics cannot explain the difference between the effectiveness of the WIOC and WC formulations. This leaves to the favorable microenvironment created for T. ovalisporum by the WIOC formulation as the primary contributor to the formulation's improved control of frosty pod rot.

\section{Acknowledgement}

References to a company and/or product by the USDA are only for the purpose of information and do not imply approval 
or recommendation of the product to the exclusion of other that may also be suitable. USDA is an equal opportunity provider and employer.

\section{Funding Details}

This work was supported by the USDA, Agricultural Research Service under CRIS [8042-21000-278-00D].

\section{Conflict of Interest}

This is to acknowledge no financial interest or benefit has arisen from the direct applications of this research.

\section{References}

1. Crozier J, Arroyo C, Morales H, Melnick RL, Strem MD, et al. (2015) The influence of formulation on Trichoderma biological activity and frosty pod rot management in Theobromo cacao. Plant pathology 64(6): 13851395.

2. Boyette CD (1994) Unrefined corn oil improved the mycoherbicidal activity of Collectotrichum truncatum for hemp sesbania (Sesbania exaltata) control. Weed Technology 8(3): 526-9.
3. Boyette CD, Hoagland RE, Weaver MA (2007) Biocontrol efficacy of Colletotrichum truncatum for hemp sesbania (Sesbania exaltata) is enhanced with unrefined corn oil and surfactant. Weed Biology and Management 7: 70-76.

4. Mbarga JB, Ten Hoopen GM, Begoude AD, Tondje PR, Juate J, et al. (2012) Efficacy of Trichoderam asperellum Oil Formulations on the Control of Cocoa Blcack Pod Disease (Phytophtora megakarya). Comm App Biol Sci, Ghent University, pp. 65-73.

5. Clark J (2013) Emulsions: When Oil and Water Do Mix. Food Technology 67(8):1-8.

6. Zhu H, Salyani M, Fox M (2011) A Portable scanning system for evaluation of spray deposit distripution. Computers and Electronics in Agriculture 76(1): 38-43.

7. Schick RJ (2002) Spray Technology Reference Guide. Chads Ford, PA: Spraying Systems Co.

8. SAS Institute Inc (2015) SAS/StAT 14.1 Users's Guide. Cary, NC: SAS Institute Inc. 